\title{
COMPARISON OF SINGLE-HOP AND MULTIHOP AWG BASED WDM NETWORKS*
}

\author{
Hagen Woesner, Martin Maier and Adam Wolisz \\ TU Berlin, Telecommunication Networks Group (TKN), \\ Einsteinufer 25, 10587 Berlin, Germany \\ \{woesner, maier, wolisz\} @ ee.tu-berlin.de
}

\begin{abstract}
In this paper, we discuss and compare the architectures of Arrayed-Waveguide Grating (AWG) based singlehop and multihop WDM networks. We present expressions and tight upper and lower bounds for the mean hop distance and capacity of the singlehop and multihop WDM networks. Guidelines are provided for the design of AWG-based WDM networks with different population, capacity, and cost requirements.
\end{abstract}

Keywords: WDM, AWG, single-hop network, multihop network

\section{Introduction}

An increasing number of challenges encountered in optical Wavelength Division Multiplexing (WDM) networks can be met by deploying the ArrayedWaveguide Grating (AWG). Apart from simple wavelength multiplexers and/or demultiplexers, AWGs have been used to realize network elements such as Optical Add-Drop Multiplexers (OADMs) or dispersion slope compensators. In the last few years WDM networks using the AWG as a passive wavelength router have attracted much attention. Due to spatial wavelenth reuse AWG-based WDM networks are highly efficient. These networks range from LANs [2] and Passive Optical Networks (PONs) [5] to national-scale networks [3].

Recently, some AWG-based architectures geared towards the future high-profit metro WDM market were reported [4], [7]. AWG-based metro WDM networks come in two flavors: Single-hop [6] and multihop networks [8]. In this paper, we address the fundamental question whether single-hop or multihop AWGbased WDM networks provide a better performance for a given number of nodes and financial budget. For our comparison we consider a completely passive net-

* This work was done within the TransiNet project supported by the Federal German Ministry of Education and Research. 


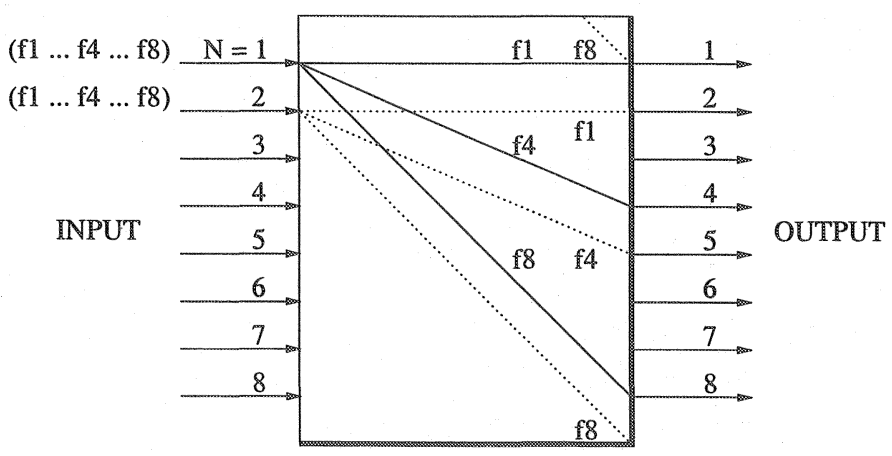

Figure 1. Wavelength routing pattern of an $8 \times 8 \mathrm{AWG}$

work which consists of a single AWG. This is reasonable since metro networks are rather cost-sensitive. Thus, the overall network costs are mainly caused by the structure of the nodes attached to the AWG. Note that not only the type of transceiver - tunable vs. fixed-tuned - and the number of transceivers used at each node determine the costs but also other aspects such as power consumption and management.

The remainder of the paper is organized as follows. In Section 2 we explain the key properties of an AWG and describe both single-hop and multihop architectures. The mean hop distance of both networks is calculated in Section 3. In Section 4 we compare the networks in terms of aggregate capacity. We present some illustrative results in Section 5 and conclude in Section 6.

\section{Arrayed-Waveguide Grating}

The Arrayed-Waveguide Grating (AWG) is a passive optical wavelength router. Using one Free Spectral Range (FSR) an $N \times N$ AWG simultaneously accepts at each input port a total of $N$ wavelengths and routes each wavelength to a particular output port without resulting channel collisions, i.e., the AWG is a strictly nonblocking device. At each output port arrive $N$ wavelengths, one from each input port. As a consequence, a receiver attached to an AWG output port has to be tunable over a range of $N$ wavelengths in order to obtain data from all AWG input ports. There exist cyclic wavelength permutations at the output ports if different input ports are used. The routing pattern of an $8 \times 8$ AWG is depicted in Fig. 1. The output ports are numbered in the same direction that increasing frequency values are diffracted by the AWG. Each optical frequency (wavelength) gives routing instructions that are independent of the AWG input port. Thus, frequency $k$ 's routing information is to exit the output port that is $(k-1)$ ports below the corresponding input port, i.e., $\mathrm{f} 1$ goes from input port 1 to exit port 1 and from input 5 to exit port 5 . Similarly, $f 3$ incident 
on input port 1 is directed to output port 3 , whereas if $\mathrm{f} 3$ were incident on port 5 it would be routed to output port 7 . Note that the AWG is a cyclic wavelength router. Consequently, f8 launched into input port 2 is routed to AWG output port 1, as illustrated in Fig. 1. In general, fi entering at input port $j$ will exit at output port $k$, where $k=((N+i+j-2) \bmod N)+1$ with $N \geq 2$ and $i, j \in\{1,2, \ldots, N\}$.

Next, let us consider WDM networks that are based on an AWG. More specifically, $N$ nodes are interconnected by an $N \times N$ AWG such that the transmitting part of a node is attached to one of the $N$ AWG input ports and the receiving part of that node is located at the opposite AWG output port. If a given node is able to transmit on all $N$ wavelengths - either by using an array of $N$ fixedtuned transceivers each operating on a different wavelength or by deploying one or more tunable transceivers - all nodes (including the source node) can be reached in one hop resulting in a single-hop network. However, equipping each station with multiple tunable transceivers or $N$ fixed-tuned transceivers might be economically prohibitive. A reasonable cost-performance tradeoff might be achieved by using $r$ fixed-tuned transceivers at each node, where $1 \leq r \leq(N-1)$. Generally, this implies that a given packet has to traverse multiple intermediate nodes along its path to the destination node leading to multihop networks. Note that $r=(N-1)$ transceivers are sufficient to realize a single-hop network since in general no node needs to send packets to itself.

\subsection{Single-Hop Architecture}

In the single-hop network architecture each node has one or more tunable transceiver. The case where alternatively every node is equipped with an array of $(N-1)$ fixed-tuned transceivers is considered a special case of multihop networks with $r=(N-1)$ and will be discussed later. For communicating with different nodes each node has to tune its transceivers to different wavelengths. The incurred tuning latency largely depends on the type of transceiver in use. Table 1 shows the tuning range and tuning time of electro-optic, acousto-optic, and mechanically tunable transceivers, respectively. The required tuning range is determined by the number of used wavelengths $(N-1)$ and the channel spacing. Note that the tuning times of the various transceiver types differ by multiple orders of magnitude. Hence, for a given channel spacing the parameter $N$ has a strong impact on the tuning overhead.

\subsection{Multihop Architecture}

A multihop network using the AWG in a physical star architecture can be constructed in the following way: Assume that node $A$ is connected to the first input/output pair, node B to the second one, and so on. Each node is equipped with one or more transceivers, each fixed-tuned to a different wavelength. As 
Table 1. Transceivers: Tuning Ranges and Tuning Times

\begin{tabular}{lll}
\hline Transceiver Type & Tuning Range & Tuning Time \\
\hline Electro-optic & $10-15 \mathrm{~nm}$ & $1-10 \mathrm{~ns}$ \\
Acousto-optic & $\sim 100 \mathrm{~nm}$ & $\sim 10 \mu \mathrm{s}$ \\
Mechanically tunable & $500 \mathrm{~nm}$ & $1-10 \mathrm{~ms}$ \\
\hline
\end{tabular}

explained in Section 2, wavelength $\lambda_{1}$ is always routed back to the node where it came from, so it can not be used for the transmission to other nodes. But the other $(N-1)$ wavelengths may be seen as virtual rings. For a $5 \times 5 \mathrm{AWG}$ network this is shown in Fig. 2. Note that the virtual rings on wavelength $\lambda_{2}$ and $\lambda_{5}$ are counterdirectional as well as $\lambda_{3}$ and $\lambda_{4}$. The resulting connectivity pattern can be considered a fully meshed interconnection. It can be seen that potentially all wavelengths can be used for transmission between a given pair of nodes.

However, there might be wavelengths which can not be used for transmission to all nodes. This is shown for a $4 \times 4$ AWG in Fig. 3, where $\lambda_{3}$ makes up two separate rings ( $A-C$ and $B-D)$ which are node disjoint resulting in logically disjoint subnetworks. Note that these subnetworks exist in all AWG-based multihop networks where the AWG degree is not a prime number. Only with $N$ being a prime number the network consists of $(N-1)$ virtual rings with all nodes connected to all rings (for more information please refer to [8]).

In general, there is no need to have as many transmitter/receiver-pairs (transceivers) as wavelengths in the system. It is possible to start with only one fixed-tuned transceiver per node, which results in a unidirectional ring (e.g., using only $\lambda_{2}$ in Fig. 2). Intuitively, equipping each node with additional transceivers, each fixed tuned to a separate wavelength, decreases the hop distance between nodes

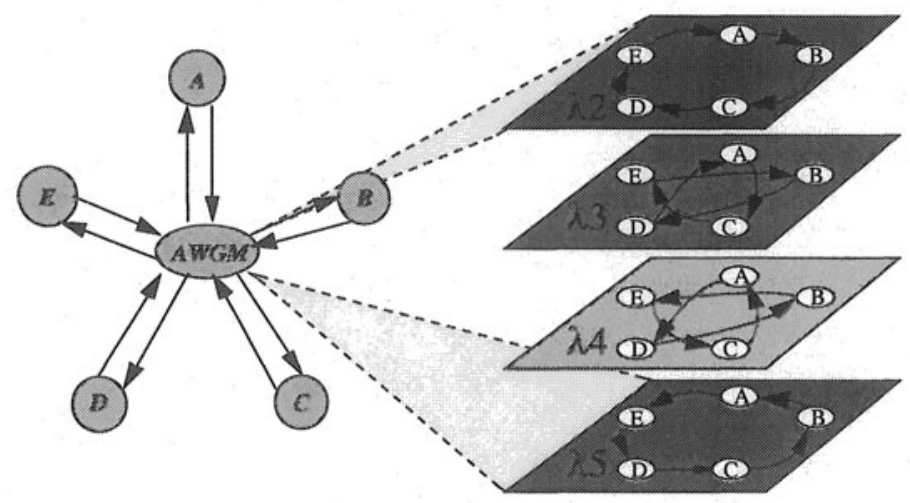

Figure 2. Connections in a network of 5 nodes using 4 wavelengths. 


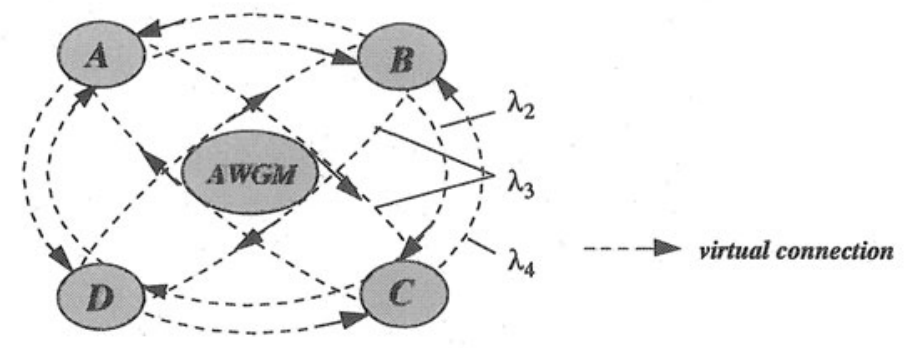

Figure 3. Basic vitual topology of a network made up by a $4 \times 4$ AWG. For better visibility only virtual connections are shown.

and increases the achievable throughput of each node. Thereby the available bandwidth between two endpoints can be scaled up to the actual needs. An analysis of the available capacity follows in section 4 after calculating the mean hop distance in the following section.

\section{Mean Hop Distance}

Let one hop denote the distance between two logically adjacent nodes. The mean hop distance denotes the average value of the minimum numbers of hops a data packet has to make on its shortest way from a given source node to all remaining $(N-1)$ destination nodes. Note that in both single-hop and multihop networks the mean hop distance is the same for all (source) nodes. In the following we assume uniform traffic, i.e., a given source node sends a data packet to any of the resulting $(N-1)$ destination nodes with equal probability $1 /(N-1)$.

\subsection{Single-hop Network}

Clearly, in the single-hop network each source node can reach any arbitrary distination node in one hop. Thus, the mean hop distance is given by

$$
\bar{h}_{S}=1
$$

\subsection{Multihop Network}

For illustration, let us begin with the simple case where we use only one wavelength such that we obtain a unidirectional ring. The mean hop distance is then given by

$$
\bar{h}=\frac{1}{N-1} \sum_{i=1}^{N-1} i=\frac{N(N-1)}{2(N-1)}=\frac{N}{2}
$$


The distance between an initial node and the other nodes is equal to $1,2, \ldots,(N-$ 1), respectively. That is, we walk around the ring. Next, let us deploy an additional wavelength. Adding another ring should decrease the mean hop distance as much as possible. To do so, the additional wavelength has to be chosen such that the second ring is counterdirectional to the first one already in use. Consequently, for odd $N$ we would walk $1,2, \ldots,(N-1) / 2$ hops in each direction. The resulting mean hop distance is given by

$$
\bar{h}=\frac{1}{N-1} \frac{2\left(\frac{N-1}{2}+1\right) \frac{N-1}{2}}{2}=\frac{N+1}{4}
$$

Note that in Equ.(3) and in the following we assume that wavelength conversion is not supported at any node. Again, this is reasonable since our networks are intended to provide cost-effective metro solutions. (By allowing for wavelength conversion a smaller mean hop distance could be achieved.)

For the generic calculation of the mean hop distance let the parameter $1 \leq r_{M} \leq$ $(N-1)$ denote the number of simultaneously used wavelengths (transceivers) at each node. The mean hop distance $\bar{h}_{M}$ of the resulting multihop network is lower bounded by

$$
\begin{aligned}
\bar{h}_{M} & \geq \sum_{h=1}^{\left.\frac{N-1}{r_{M}}\right\rfloor} \frac{r_{M}}{N-1} \cdot h+\frac{(N-1) \bmod r_{M}}{N-1} \cdot\left\lceil\frac{N-1}{r_{M}}\right\rceil \\
& =\frac{1}{N-1}\left\{r_{M} \frac{\left\lfloor\frac{N-1}{r_{M}}\right\rfloor\left(\left\lfloor\frac{N-1}{r_{M}}\right\rfloor+1\right)}{2}+\left[(N-1) \bmod r_{M}\right]\left\lceil\frac{N-1}{r_{M}}\right\rceil\right\}
\end{aligned}
$$

To see this, note that the mean hop distance becomes minimum if (1) as many different nodes as possible are reached in each hop count starting with one hop and (2) the maximum hop distance (diameter) of the network is minimum. Applying this leads us to Equ. (4). Since a given source node sends on $r_{M}$ wavelengths at most $r_{M}$ different destination nodes can be reached for each hop count. Each time exactly $r_{M}$ different destination nodes are reached up to a hop count of $\left\lfloor\frac{N-1}{r_{M}}\right\rfloor$, which corresponds to the first term of Equ.(4). The second term of Equ. (4) counts for the remaining nodes (less than $r_{M}$ ) which are $\left\lceil\frac{N-1}{r_{M}}\right\rceil$ hops away from the given source node.

Next, we compute the the mean hop distance $\bar{h}_{M}$. For large $N$ we assume a uniform distribution of the number of hops to every station over all rings. (Each station is reached only once in a ring, and in a different hop number for every ring.) The probability of a certain hop count to be selected equals $1 /(N-1)$. 
The probability of a certain hop number $h$ to be the minimum of all selected rings $r_{M}$ is the probability that the hop number is selected by one of the $r_{M}$ rings times the probability that all the remaining $\left(r_{M}-1\right)$ rings have a hop number between $h$ and $(N-1)$. Of course, if the remaining area is smaller than the number of the remaining rings, the probability of this $h$ to be the minimum is zero:

$$
p\left(h_{m i n}\right)=\left\{\begin{aligned}
\frac{r_{M}\left(\begin{array}{c}
(N-1-h) \\
\left(r_{M}-1\right)
\end{array}\right)}{(N-1)\left(\begin{array}{l}
\left(N_{M}-1\right) \\
(N-1)
\end{array}\right)}: & h<=N-r_{M} \\
0 & : h>N-r_{M}
\end{aligned}\right.
$$

The mean hop distance $\bar{h}_{M}$ is equal to the expected value of $h_{\min }$ :

$$
\bar{h}_{M}=E\left[h_{m i n}\right]=\frac{1}{N-1} \sum_{N=1}^{N-1} \sum_{h=1}^{N-r_{M}} h p\left(h_{m i n}\right)
$$

where the addition of the $\bar{h}$ over all stations can be omitted since we assume $\bar{h}$ to be the same for every station. Thus, we get

$$
\begin{aligned}
\bar{h}_{M}=E\left[h_{m i n}\right] & =\sum_{h=1}^{N-r_{M}} h \frac{r_{M}}{N-1} \frac{\left(\begin{array}{c}
(N-1-h) \\
\left(r_{M}-1\right)
\end{array}\right)}{\left(\begin{array}{c}
(N-2) \\
\left(r_{M}-1\right)
\end{array}\right)} \\
& =\frac{N^{2}\left(\begin{array}{c}
N-1 \\
r_{M}
\end{array}\right)}{\left(r_{M}+1\right)\left(N-r_{M}\right)\left(\begin{array}{c}
N \\
r_{M}
\end{array}\right)} \\
& =\frac{N^{2}(N-1) !\left(N-r_{M}\right) !}{\left(N-1-r_{M}\right) !\left(r_{M}+1\right)\left(N-r_{M}\right) N !} \\
& =\frac{N^{2}(N-1) !\left(N-r_{M}\right) !}{\left(r_{M}+1\right) N(N-1) !\left(N-r_{M}\right) !}
\end{aligned}
$$

which surprisingly boils down to

$$
\bar{h}_{M}=\mathbb{E}\left[h_{m i n}\right]=\frac{N}{r_{M}+1} .
$$

Equ.(12) gives the mean hop distance for all possible combinations of $r_{M}$ rings. Therefore, it is an upper bound for the mean hop distance of the best choice of the wavelengths. The choice of the wavelength (=ring) to add depends on the resulting mean hop distance. The problem of the right choice seems to be NP-hard, although we can not prove this up to now. It seems to be of the family of knap-sack (or Rucksack) problems. Definitely, it is not the best idea to always go and look for counterdirectional rings. For instance, in the case of 
Table 2. Mean hop distances for optimum combinations of wavelengths in multihop networks

$\begin{array}{lccccc}\text { No. of nodes } N & 3 & 5 & 7 & 11 & 13 \\ \text { No. of rings } r_{M} & & & & & \\ 1 & 1.5 & 2.5 & 3.5 & 5.5 & 6.5 \\ 2 & 1.0 & 1.5 & 2.0 & 3.0 & 3.5 \\ 3 & & 1.25 & 1.67 & 2.5 & 2.92 \\ 4 & & 1.0 & 1.33 & 2.0 & 2.25 \\ 5 & & & 1.17 & 1.5 & 1.75 \\ 6 & & & 1.0 & 1.4 & 1.5 \\ 7 & & & & 1.3 & 1.42 \\ 8 & & & & 1.2 & 1.33 \\ 9 & & & & 1.1 & 1.25 \\ 10 & & & & 1.0 & 1.17 \\ 11 & & & & & 1.08 \\ 12 & & & & & 1.0\end{array}$

$N=13$ and $r_{M}=4$, the combination of any two pairs of counterdirectional rings like $[1,4,9,12]$ leads to a mean hop distance of $\bar{h}_{M}=\frac{7}{3}=2.3 \overline{3}$ while $\bar{h}_{M}=\frac{9}{4}=2.25$ for a combination of rings $[1,4,6,11]$. But a choice of the next ring to be counterdirectional to the previous one seems to be a good heuristic and is in most cases near to the optimum value.

Table 2 shows the mean hop distances of multihop networks for prime $N$ up to $N=13$. These were obtained through exhaustive search by taking the best (smallest mean hop distance) of all possible combinations of rings for a given value of $r_{M}$ and $N$, respectively. Fig. 4 depicts the lowest achievable mean hop distance as a function of $r_{M}$ for $N=11$. Apparently, increasing $r_{M}$, i.e., adding fixed-tuned transceivers to each node decreases the mean hop distance. The minimum mean hop distance equals one and is achieved for $r_{M}=N-1=10$. Note that the lower bound of the mean hop distance is tight. For the other values of $N$ presented in Table 2 we observed that the upper bound is tight as well.

\section{Performance Comparison}

Beside the mean hop distance the single-hop and multihop networks are compared in terms of network capacity. According to [1], let the network capacity $C$ be defined as

$$
C=\frac{r \cdot S \cdot N}{\bar{h}}
$$

where $r$ denotes the number of transceivers per node, $S$ stands for the transmitting rate of each transmitter, $N$ represents the number of nodes in the network, and $\bar{h}$ denotes the mean hop distance of the network. 


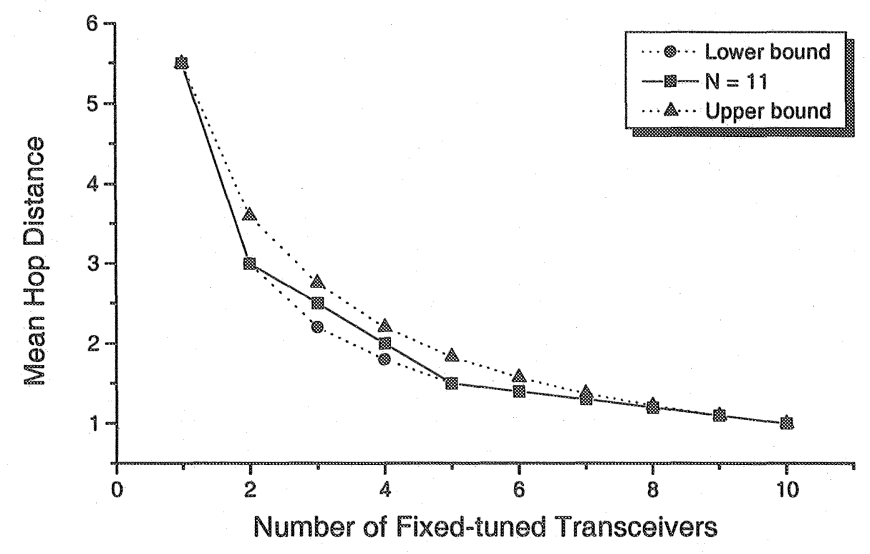

Figure 4. Mean hop distance of a multihop network vs. $r_{M}$ for $N=11$.

\subsection{Single-hop Network}

As mentioned in Section 2.1, in the single-hop network each node is equipped with $r_{S}$ tunable transceivers, where $r_{S} \geq 1$. We consider fixed-size packets and assume that the transceiver has to be tuned to another wavelength after transmitting a data packet (we thereby provide a conservative capacity evaluation). Due to the nonzero tuning time $\tau$ of the transceiver the effective transmitting rate is decreased as follows

$$
\begin{aligned}
S_{S} & =\frac{L}{L+\tau} \cdot S \\
& =\frac{1}{1+\tau_{L}} \cdot S, \tau_{L}=\frac{\tau}{L}
\end{aligned}
$$

where $\tau_{L}$ denotes the transceiver tuning time normalized by the packet transmission time $L$. With $\bar{h}_{S}=1$ the capacity of the single-hop network equates to

$$
C_{S}=\frac{r_{S} \cdot S \cdot N}{1+\tau_{L}}
$$




\subsection{Multihop Network}

In the multihop network $r$ is equal to the number of used wavelengths (transceivers) $r_{M}$ as explained in section 3.2, i.e., $r=r_{M}$ where $r_{M}=$ $1,2, \ldots,(N-1)$. Since the transceivers are fixed-tuned there is no tuning penalty. Consequently, the effective transmitting rate equals $S$. Using the upper bound of the mean hop distance given in Equ.(12) we get the lower bound of the capacity as follows

$$
C_{M} \geq \frac{r_{M} \cdot S \cdot N}{\bar{h}_{M \max }}=r_{M} \cdot\left(r_{M}+1\right) \cdot S .
$$

Similarly, using the lower limit of the mean hop distance given in Equ.(5) conveys the upper bound of the capacity

$$
\begin{aligned}
C_{M} \leq & \frac{r_{M} \cdot S \cdot N}{\bar{h}_{M_{\text {min }}}} \\
= & \frac{r_{M} \cdot S \cdot N \cdot(N-1)}{r_{M} \frac{\left\lfloor\frac{N-1}{r_{M}}\right\rfloor\left(\left\lfloor\frac{N-1}{r_{M}}\right\rfloor+1\right)}{2}+\left[(N-1) \bmod r_{M}\right]\left\lceil\frac{N-1}{r_{M}}\right\rceil}
\end{aligned}
$$

Next, we want to calculate the proportion of fixed-tuned transceivers that has to be deployed to achieve the same network capacity as a single-hop network with a given number of nodes. Therefore, we start by equating the capacities from Equs.(17) and (16):

$$
\begin{aligned}
C_{M} & =C_{S} \\
r_{M} \cdot\left(r_{M}+1\right) \cdot S & =\frac{r_{S} \cdot S \cdot N}{1+\tau_{L}} \\
r_{M}^{2}+r_{M}-\frac{r_{S} \cdot N}{1+\tau_{L}} & =0 \\
r_{M} & =-\frac{1}{2} \pm \sqrt{\frac{1}{4}+\frac{r_{S} \cdot N}{1+\tau_{L}}}
\end{aligned}
$$

Equ.(23) gives the number of fixed-tuned transceivers $r_{M}$ per node in a multihop network whose capacity is equal to that of a single-hop network with $r_{S}$ tunable transceivers at each node for a given population $N$.

\section{Numerical Results}

In all presented numerical results we consider fixed-size packets with a length of 1500 bytes and a transmitting rate of $10 \mathrm{Gbps}$. This translates into a 


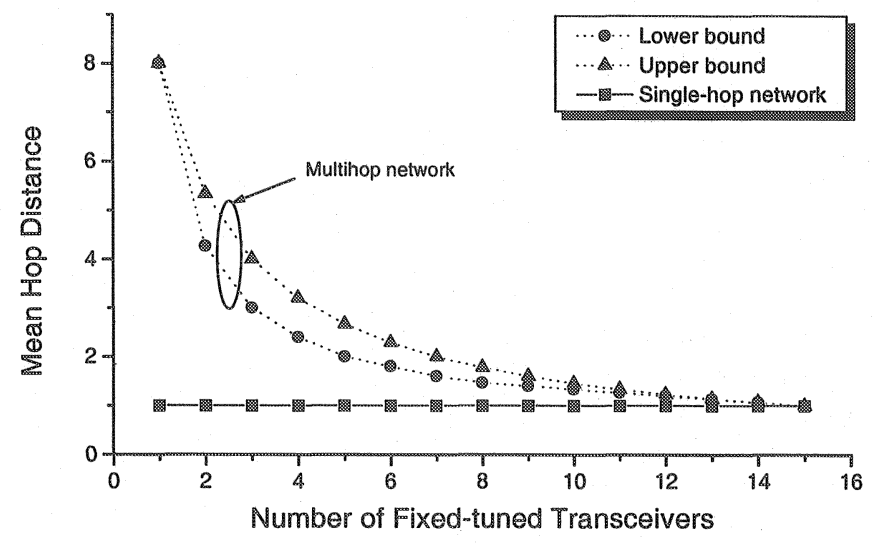

Figure 5. Mean hop distance vs. $r_{M}$ for $N=16$.

packet transmission time equal to $L=1.2 \mu \mathrm{s}$. The channel spacing is assumed to be $100 \mathrm{GHz}(0.8 \mathrm{~nm}$ at $1.55 \mu \mathrm{m})$.

First, we consider a network with a population of $N=16$ nodes. Fig. 5 illustrates that the mean hop distance of the single-hop network is one, independent of $r_{M}$. We observe that both the upper and lower bound of the mean hop distance of the corresponding multihop network decrease exponentially with increasing $r_{M}$. As a consequence, a few fixed-tuned transceivers at each node are sufficient to decrease the mean hop distance of the multihop network dramatically and to get close to the mean hop distance of the single-hop network. Adding further transceivers has only a small impact on the resulting mean hop distance. For $r_{M}=N-1=15$ both single-hop and multihop networks have the same mean hop distance, namely, one.

However, from the network capacity point of view equipping each node with as many fixed-tuned transceivers as possible is beneficial. This can be seen in Fig. 6 which depicts the network capacity (bounds) in Gbps as a function of $r_{M}$ for a multihop network in comparison to a single-hop network with $r_{S}=1$. While the network capacity of the single-hop network remains constant the capacity of the multihop counterpart quadratically increases for increasing $r_{M}$. This is due to the fact that a large $r_{M}$ not only decreases the mean hop distance but also increases the degree of concurrency by using all transceivers simultaneously. Note that the multihop network requires at least four fixedtuned transceivers per node in order to outperform its single-hop counterpart with one single tunable transceiver per node in terms of capacity. 


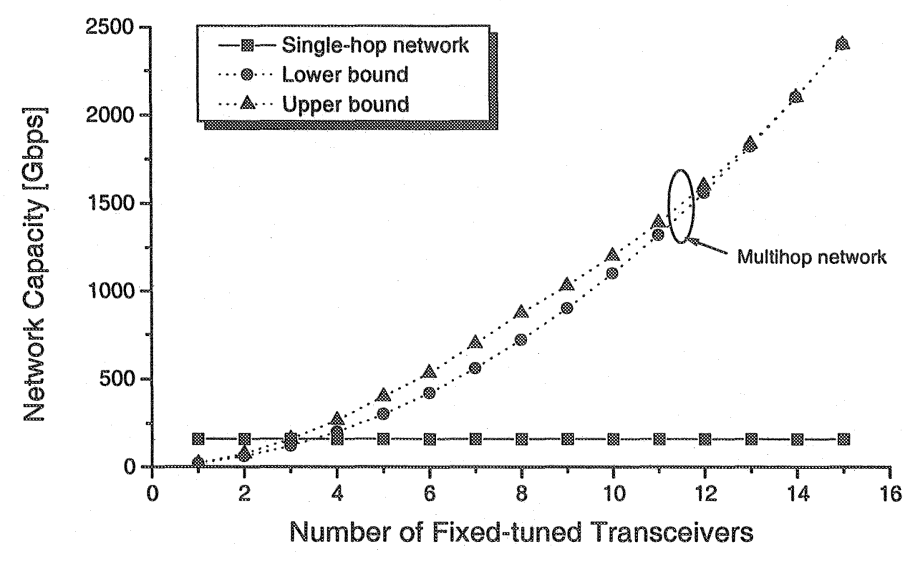

Figure 6. Network capacity vs. $r_{M}$ for $N=16$.

Recall from Section 2.1 that for a given channel spacing the number of nodes $N$ determines the required tuning range of the tunable transceivers used in the single-hop network. From Table 1 we learn that with a channel spacing of $0.8 \mathrm{~nm}$ we can deploy fast tunable electro-optical transceivers for up to $N=16$ nodes, approximately. This translates into a negligible normalized tuning time $\tau_{L}=8.3 \overline{3} \cdot 10^{-3}$. In contrast, for $N>16$ acousto-optic transceivers have to be applied which exhibit a three orders of magnitude times larger tuning time. Hence, we obtain a normalized tuning time $\tau_{L}=8.3 \overline{3}$. The impact of the transceiver tuning time on the network capacity is shown in Fig. 7, again for $r_{S}=1$. For $N \leq 16$ the capacity of the single-hop network grows linearly with $N$.

For $N>16$ acousto-optic transceivers have to be applied instead of electrooptic ones. The incurred larger tuning time dramatically decreases the network capacity. For increasing $N$ the network capacity again grows linearly but the slope is smaller.

In addition, Fig. 7 depicts the lower capacity bound of the multihop network. Interestingly, this bound remains constant for varying $N$. This is because with increasing $N$ more nodes contribute to the network capacity but each node has to forward packets for a larger fraction of time due to the increased mean hop distance resulting in a lower net data rate per node. Equ.(17) reflects this point more precisely; both the number of transmitting nodes and the mean hop distance are directly proportional to $N$ such that the lower capacity bound is independent of $N$. 


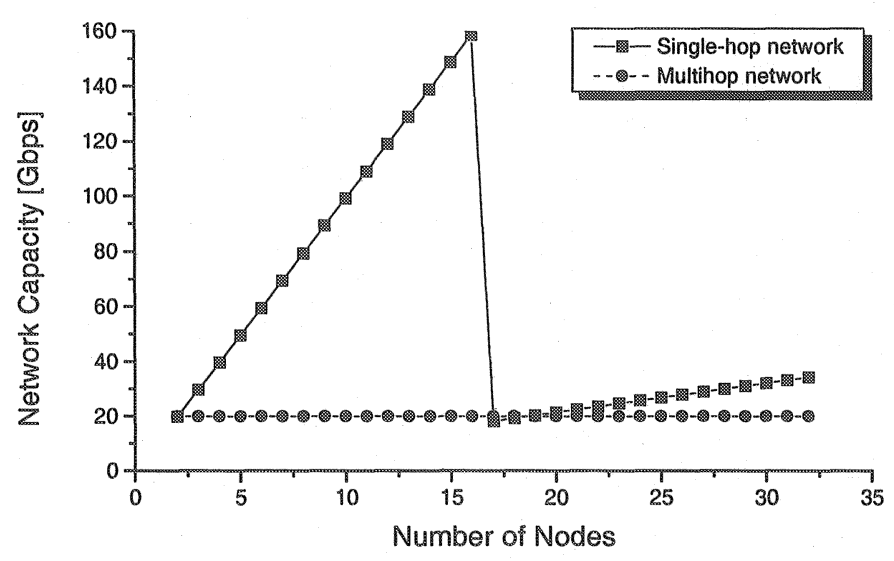

Figure 7. Network capacity vs. $N$.

The dependency of $r_{M}$ on $N$ and $r_{S}$ was calculated in Equ.(23). It is shown in Fig. 8. The z-axis depicts the number of fixed-tuned transceivers $r_{M}$ that must replace one tunable transceiver in order to achieve the same network capacity in both multihop and single-hop networks. Note that this graph can also be used to help decision makers design an appropriate AWG-based WDM network - either single-hop or multihop - for given population, capacity, and cost scenarios, as outlined in the following section.

\section{Conclusion}

WDM networks based on an AWG are either single-hop or multihop networks. In the single-hop network each node has (at least) one tunable transceiver. Equipping each node with one or more fixed-tuned transceivers results in multihop networks that consist of multiple virtual rings. We have compared both single-hop and multihop networks in terms of mean hop distance and aggregate capacity. Moreover, we have addressed the question which network type provides a higher capacity for a given number of nodes. The answer to this question largely depends on the cost ratio of fixed-tuned and tunable transceivers and on the tuning latency of the tunable transceivers. Fig. 8 can be interpreted such that it shows the relation of the cost of a tunable transceiver unit to the cost of a fixed transceiver unit. A unit comprises not only the respective transceiver but all additional items that are required for proper operation. Generally, these include initial capital expenditure, operation, management, and 


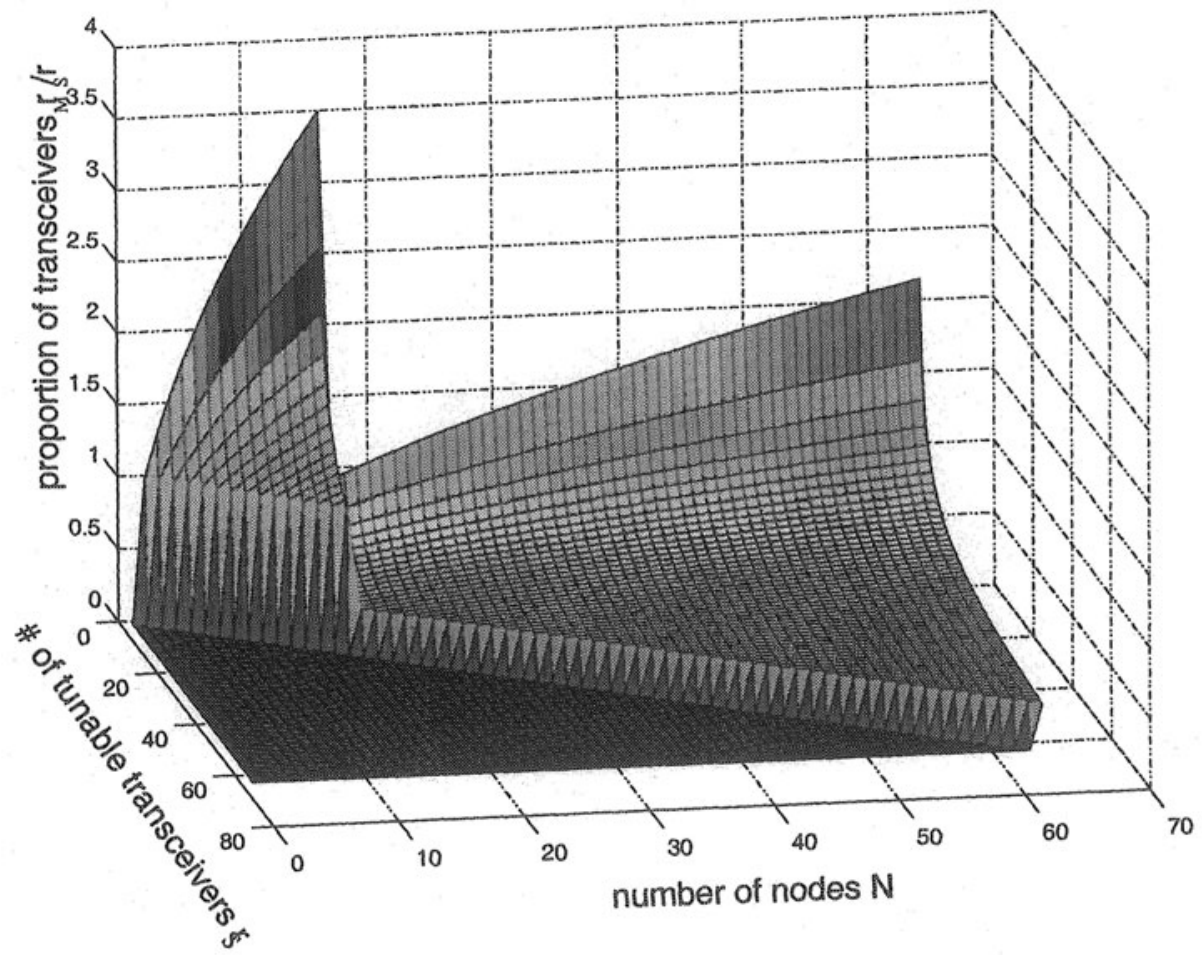

Figure 8. Proportion $r_{M} / r_{S}$ of fixed-tuned to tunable transceivers that is needed to achieve the same network capacity in a single--hop network with $r_{S}$ tunable transceivers and in a multihop network with $r_{M}$ fixed-tuned transceivers per node. 
maintenance costs. Fig. 8 illustrates that for interconnecting $N=16$ nodes in a multihop architecture around 3.51 (that is, at least four) fixed transceiver units should replace one tunable transmitter (this can also be observed in Fig. 6). If, however, the desired capacity should be four times as large, requiring 4 tunable transceivers, the relation reduces to around 1.8 , meaning that 8 fixed transceivers would be sufficient per node. It can be seen from this that not just the price of the components should influence the choice of the network architecture, but also the number of nodes and the desired network capacity.

Clearly, many more factors add to the price of the single transmitter and receiver component. For instance, wavelength multiplexers and demultiplexers are needed before and after fixed transceivers, respectively. Optical splitters and combiners would be used in the same place when applying a single-hop architecture. In addition, we note that the cost comparison of AWG-based single-hop vs. multihop networks has also to take the power budget into account. Since in the multihop networks packets have to pass the AWG multiple times optical amplifiers (EDFAs) might be mandatory. The resulting costs had to be added to the costs of the fixed-tuned transceiver.

It should be noted, however, that the figures provided here are independent of the actual cost of the transmitter units, but instead should serve as a general guideline for the choice of the proper network architecture.

\section{References}

[1] A. S. Acampora and S. I. A. Shah. Multihop lighwave networks: A comparison of storeand-forward and hot-potato routing. In Proc., IEEE INFOCOM, pages pp. 10-19, 1991.

[2] M. S. Borella, J. P. Jue, and B. Mukherjee. Simple scheduling algorithms for use with a waveguide grating multiplexer based local optical network. Photonic Network Commun., 1(1), 1999.

[3] N. P. Caponio, A. M. Hill, F. Neri, and R. Sabella. Single-layer optical platform based on WDM/TDM multiple access for large-scale 'switchless' networks. European Trans. on Telecommunications, 11(1):73-82, 2000.

[4] K. Kato, A. Okada, and et al. Sakai, Y. 10-tbps full-mesh WDM network based on cyclicfrequency arrayed-waveguide grating router. In Proc. of ECOC 2000, volume 1, pages 105-107, 2000.

[5] G. Maier, M. Martinelli, A. Pattavina, and E. Salvadori. Design and cost performance of the multistage WDM-PON access networks. IEEE/OSA J. of Lightwave Technology, 18(2):pp. 125-143, 2000.

[6] M. Maier, M. Reisslein, and A. Wolisz. High-performance switchless WDM network using multiple free spectral ranges of an arrayed-waveguide grating. In Proc., Terabit Optical Networking: Architecture, Control, and Management Issues, Part of SPIE Photonics East, pages 101-112, 2000.

[7] F. Ruehl and T. Anderson. Cost-effective metro WDM network architectures. In OFC 2001 Technical Digest, paper WLI, 2001.

[8] H. Woesner. Primenet - network design based on arrayed waveguide grating multiplexers. In Proc., SPIE Design and Manufacturing of WDM Devices, pages 22-28, 1998. 